\title{
Órganos Abdominales: Peso del Hígado, Bazo y Riñones en Relación a Sexo, Grupo Étnico, Edad e Índice de Masa Corporal en Individuos Chilenos
}

\author{
Abdominal Organs: Weight of the Liver, Spleen and Kidneys in Relation to Sex, \\ Ethnic Group, Age and Body Mass Index in Chilean Individuals
}

Olave, E.*; Torrez, J. C."; Riquelme, N.**; Ibacache, L.** \& Binvignat, O.***

OLAVE, E.; TORREZ, J. C.; RIQUELME, N.; IBACACHE, L. \& BINVIGNAT, O. Órganos abdominales: Peso del hígado, bazo y riñones en relación a sexo, grupo étnico, edad e índice de masa corporal en individuos chilenos. Int. J. Morphol., 32(4):1311-1315, 2014.

RESUMEN: El aumento del peso de los órganos puede ser signo de enfermedades o anormalidades patológicas. Diversos estudios han llevado a la elaboración de tablas con los pesos estándar de los diferentes órganos en diversos grupos poblacionales. Basados en esta premisa, determinamos el peso del hígado, bazo y riñones en dos grupos de individuos Chilenos, Mapuches (M) y NoMapuches (n-M), de ambos sexos y relacionamos los resultados con la edad e índice de masa corporal (IMC). El peso promedio del hígado en el hombre, con relación a la edad, aumenta levemente (n-M) o disminuye en un pequeño porcentaje (M). Sin embargo, en la mujer (M) el peso aumentó en un 22,8\% En relación al IMC, el peso promedio del hígado, aumenta en los hombres de ambos grupos étnicos en alrededor de un tercio del peso registrado en personas con IMC de 20-25; en mujeres de ambos grupos el peso disminuye levemente. El peso del bazo, en relación a la edad, disminuye alrededor de un 4\%, en hombres de ambos grupos étnicos y mujeres (n-M) a diferencia de las mujeres (M) que se incrementa en un 18\%. En relación al IMC, el peso del bazo aumenta en los hombres de ambos grupos étnicos en alrededor de un $40 \%$. Los riñones tienen un leve incremento en su peso promedio en ambos grupos. De acuerdo al IMC, el peso de los riñones aumenta en hombres de ambos grupos. En las mujeres (M) el peso se mantiene o disminuye levemente; en las mujeres (n-M) ambos riñones incrementan su peso. Los datos aportados en esta investigación servirán de referencia a la anatomía clínica y quirúrgica de los órganos abdominales de nuestra población.

PALABRAS CLAVE: Anatomía; Abdomen; Peso; Hígado; Bazo; Riñones.

\section{INTRODUCCIÓN}

El aumento del peso de los órganos puede ser signo de enfermedades o anormalidades patológicas. La literatura muestra diferentes estudios que han llevado a la elaboración de tablas con los pesos estándar de los diferentes órganos en diversos grupos poblacionales.

Dentro de esos estudios, Molina \& DiMaio (2012) determinaron en un grupo de individuos fallecidos súbitamente y de forma traumática el peso del hígado, bazo y riñones, donde el primero tuvo un promedio de $1.561 \mathrm{~g}$, el segundo de $139 \mathrm{~g}$ y los últimos de $129 \mathrm{~g}$ el derecho y $137 \mathrm{~g}$, el izquierdo, realizando asociaciones con diverso parámetros tales como peso y altura del cuerpo, y por ende, con el índice de masa corporal. Estos valores son inferiores a los informados por autores como Testut \& Latarjet (1969), excepto el peso del hígado, y a los descritos por Gardner et al. (1977) y Williams et al. (1995).

Otros estudios han determinado que la edad es un factor que influye en el del peso de algunos órganos (Sheikhazadi et al., 2010; Prakash et al., 2013), así como el peso corporal (Mathuramon et al., 2009; Yi-Suk Kim et al., 2009). Otros han señalado que este último factor no incidiría en el peso de estos órganos (Molina \& Di Maio).

Vistas estas diferencias en la literatura, se realizó un estudio para determinar el peso del hígado, bazo y ri-

\footnotetext{
Facultad de Medicina, Universidad de La Frontera, Temuco, Chile

** Universidad Mayor, Temuco, Chile.

*** Universidad Autónoma de Chile, Sede Talca, Talca, Chile.
} 
ñones en dos grupos de individuos del Sur de Chile, uno correspondiente al grupo étnico Mapuche y el otro a un grupo No-Mapuche, así como asociar este parámetro con el sexo, edad y el índice de masa corporal.

\section{MATERIAL Y MÉTODO}

El estudio consideró el análisis de 775 fichas de necropsias de individuos adultos, chilenos, de ambos sexos, de los cuales 205 eran descendientes de la etnia Mapuche. Las necropsias fueron realizadas entre los años 2009 y 2013. Se consideró el peso del hígado, bazo y riñones asociando este parámetro al sexo, grupo étnico, edad, e índice de masa corporal, calculando estadísticamente el promedio, la desviación estándar, así como promedios máximos y mínimos.

\section{RESULTADOS}

En el grupo No-Mapuche el hígado tuvo un peso promedio de $1.787 \mathrm{~g}$ en el hombre y $1.569 \mathrm{~g}$ en la mujer; el bazo $135,2 \mathrm{~g}$ y $123,7 \mathrm{~g}$, en hombres y mujeres, respectivamente; el riñón derecho 153,5 g y 124 g, en hombres y mujeres, respectivamente y el riñón izquierdo $157,6 \mathrm{~g}$ en el hombre y $126,5 \mathrm{~g}$, en la mujer.

En el grupo Mapuche, el peso promedio del hígado fue $1.754 \mathrm{~g}$ en hombre y de $1.426 \mathrm{~g}$ en mujeres; el bazo tuvo un peso de $106 \mathrm{~g}$ and $91,5 \mathrm{~g}$ en hombre y mujeres, respectivamente; en hombres el riñón derecho pesó en promedio $146,2 \mathrm{~g}$ y el izquierdo $147,8 \mathrm{~g}$ y en las mujeres el peso fue de $120,3 \mathrm{~g}$ el derecho y $121,2 \mathrm{~g}$, el izquierdo.

Los promedios y desviación estándar obtenidos de los órganos mencionados, agrupados por sexo, grupo étnico e índice de masa corporal, se muestran en Tablas I a VI.

Tabla I. Peso del Hígado y Riñones en hombres y mujeres en relación a grupos etarios en individuos No-Mapuches (en gramos).

\begin{tabular}{lrrrrrl}
\hline \multirow{2}{*}{ Edad (años) } & \multicolumn{3}{c}{ Hombres } & \multicolumn{3}{c}{ Mujeres } \\
\cline { 2 - 7 } & \multicolumn{1}{c}{ Hígado } & \multicolumn{1}{c}{ Riñón der. } & Riñón izq. & Hígado & \multicolumn{1}{c}{ Riñón der. } & Riñón izq. \\
\hline $20-29$ & $1690,9 \pm 379,9$ & $140,5 \pm 35,8$ & $146,4 \pm 37,7$ & $1572,6 \pm 332,8$ & $124,2 \pm 43,2$ & $142 \pm 24,9$ \\
$30-39$ & $1840 \pm 455,5$ & $159 \pm 43,2$ & $160,3 \pm 46,1$ & $1651,4 \pm 295$ & $125,9 \pm 15,2$ & $129,6 \pm 16$ \\
$40-49$ & $1835 \pm 392,5$ & $158,2 \pm 35,5$ & $163,8 \pm 39,4$ & $1466,4 \pm 475,2$ & $121,4 \pm 20,7$ & $121,5 \pm 21,5$ \\
$50-59$ & $1798,6 \pm 385,2$ & $156,5 \pm 42,8$ & $159,8 \pm 46,1$ & $1582 \pm 376,5$ & $124,4 \pm 26,5$ & $120,7 \pm 27$ \\
\hline
\end{tabular}

Tabla II. Peso del hígado y riñones en hombres y mujeres en relación a grupos etarios en individuos mapuches (en gramos).

\begin{tabular}{llllccc}
\hline \multirow{2}{*}{ Edad (años) } & \multicolumn{3}{c}{ Hombres } & \multicolumn{3}{c}{ Mujeres } \\
\cline { 2 - 7 } & \multicolumn{1}{c}{ Hígado } & Riñón der. & Riñón izq. & Hígado & Riñón der. & Riñón izq. \\
\hline $20-29$ & $1731,8 \pm 299,8$ & $141,2 \pm 26,7$ & $147,4 \pm 28,7$ & $1292,5 \pm 199,7$ & $113,9 \pm 27$ & $112,7 \pm 21,8$ \\
$30-39$ & $1887,8 \pm 422$ & $144,6 \pm 27,1$ & $145,8 \pm 26,7$ & $1474,3 \pm 277,1$ & $123,1 \pm 20,9$ & $130,9 \pm 21,5$ \\
$40-49$ & $1791,2 \pm 353,4$ & $150,3 \pm 33,9$ & $148,8 \pm 40,6$ & $1404,7 \pm 400,2$ & $121,3 \pm 18,7$ & $116,3 \pm 25$ \\
$50-59$ & $1679,6 \pm 331,6$ & $148,1 \pm 27$ & $149,7 \pm 33,8$ & $1586,3 \pm 398,5$ & $117 \pm 13,1$ & $117,8 \pm 13,5$ \\
\hline
\end{tabular}

Tabla III. Peso del hígado y riñones en individuos no-mapuches en relación al índice de masa corporal (IMC).

\begin{tabular}{lccccrc}
\hline \multirow{2}{*}{ Edad (años) } & \multicolumn{3}{c}{ Hombres } & \multicolumn{3}{c}{ Mujeres } \\
\cline { 2 - 7 } & Hígado & Riñón der. & Riñón izq. & Hígado & Riñón der. & Riñón izq. \\
\hline $18,1-25$ & $1642,3 \pm 330,6$ & $140,9 \pm 33$ & $145,8 \pm 34,1$ & $1501,3 \pm 389,1$ & $115 \pm 26,9$ & $122,4 \pm 21,8$ \\
$25,1-30$ & $1818,9 \pm 373,8$ & $159,7 \pm 42,5$ & $163,6 \pm 47$ & $1578,4 \pm 365,3$ & $126,8 \pm 27,5$ & $126,9 \pm 28,5$ \\
$30,1-35$ & $2056,7 \pm 389,5$ & $167,3 \pm 36,9$ & $170,1 \pm 46,1$ & $1643,5 \pm 371,1$ & $134,3 \pm 24,2$ & $130,5 \pm 21,6$ \\
$35,1-40$ & $2294,6 \pm 553,3$ & $189,2 \pm 33,7$ & $185 \pm 29,9$ & --- & --- & --- \\
\hline
\end{tabular}

Tabla IV. Peso del hígado y riñones en individuos mapuches en relación al índice de masa corporal (IMC).

\begin{tabular}{lcccccc}
\hline \multirow{2}{*}{ Edad (años) } & \multicolumn{3}{c}{ Hombres } & \multicolumn{3}{c}{ Mujeres } \\
\cline { 2 - 7 } & Hígado & Riñón der. & Riñón izq. & Hígado & Riñón der. & Riñón izq. \\
\hline $18,1-25$ & $1577,9 \pm 261,9$ & $135,4 \pm 21,3$ & $142,8 \pm 25,3$ & 1368,1 & $117,6 \pm 20,5$ & $116,1 \pm 24,2$ \\
$25,1-30$ & $1814,2 \pm 314,8$ & $146,5 \pm 27,7$ & $146,7 \pm 34$ & $1347 \pm 360,9$ & $110,5 \pm 21,9$ & $117,1 \pm 12,9$ \\
$30,1-35$ & $2039,2 \pm 392,9$ & $166,4 \pm 32,7$ & $158,6 \pm 42,8$ & --- & --- & --- \\
$35,1-40$ & --- & --- & --- & --- & --- & -- \\
\hline
\end{tabular}


Tabla V. Pesos promedio del bazo en individuos Mapuche y No Mapuche en relación a la edad (en gramos).

\begin{tabular}{lcccr}
\hline \multirow{2}{*}{ Edad (años) } & \multicolumn{2}{c}{ Mapuche } & \multicolumn{2}{c}{ No Mapuche } \\
\cline { 2 - 5 } & Hombres & Mujeres & Hombres & \multicolumn{1}{c}{ Mujeres } \\
\hline $20-29$ & $116,5 \pm 39,1$ & $77,8 \pm 23,4$ & $137,5 \pm 58,1$ & $110,1 \pm 39,2$ \\
$30-39$ & $111,8 \pm 53,4$ & $89,9 \pm 29,6$ & $142,5 \pm 76,1$ & $125 \pm 46,6$ \\
$40-49$ & $105,9 \pm 15,2$ & $92,1 \pm 57,6$ & $130,4 \pm 60,1$ & $168,9 \pm 23,6$ \\
$50-59$ & $95,5 \pm 39,4$ & $95,6 \pm 24,2$ & $131,9 \pm 59,8$ & $104 \pm 39,6$ \\
\hline
\end{tabular}

Tabla VI. Pesos promedio del bazo en individuos Mapuche y No Mapuche en relación al IMC (en gramos).

\begin{tabular}{lcccc}
\hline \multirow{2}{*}{ IMC } & \multicolumn{2}{c}{ Mapuche } & \multicolumn{2}{c}{ No Mapuche } \\
\cline { 2 - 5 } & Hombres & Mujeres & Hombres & Mujeres \\
\hline $18,1-25$ & $94,8 \pm 38,8$ & $88,1 \pm 40,9$ & $114,9 \pm 42,4$ & $139,5 \pm 39,2$ \\
$25,1-30$ & $100 \pm 44,3$ & $79,3 \pm 20,9$ & $140,4 \pm 71,6$ & $119,5 \pm 37,8$ \\
$30,1-35$ & $130,1 \pm 64,3$ & $130,9 \pm 21,5$ & $167,7 \pm 71,9$ & $115,3 \pm 43$ \\
$35,1-40$ & --- & --- & $180,5 \pm 62,8$ & --- \\
\hline
\end{tabular}

\section{DISCUSIÓN}

Los valores promedios de peso del hígado, bazo y riñones han sido previamente estudiados en varios grupos poblacionales, así como se ha descrito de forma general en los tratados clásicos de Anatomía. Autores como Testut \& Latarjet han informado que el peso de este órgano fluctúa entre 1.400 y $1.500 \mathrm{~g}$, no olvidando que el hígado en el cadáver está casi vacío de sangre. Por su parte Gardner et al. y Williams et al. han descrito valores más altos indicando el peso entre $1.400 \mathrm{y}$ $1.800 \mathrm{~g}$ en el hombre y entre 1.200 y $1.400 \mathrm{~g}$ en la mujer. En general, nuestros resultados muestran valores semejantes.

Al comparar el peso promedio del hígado en nuestra serie con las de Prakash et al., cuyo estudio fue realizado en una población de la India y Yi-Suk Kim et al. (2009), que estudiaron una población Coreana, se puede apreciar que los valores obtenidos en nuestra investigación son mucho mayores que los informados en esos estudios, ya que los primeros informaron un promedio de $1.419 \mathrm{~g}$ en el hombre y $1.204 \mathrm{~g}$ en la mujer, así como los segundos registraron $1.650 \mathrm{~g}$ en el hombre y $1.344 \mathrm{~g}$ en la mujer, menor al promedio determinado en los dos grupos estudiados en nuestra serie, alrededor de $1.750 \mathrm{~g}$ en hombres y de 1.569/1.426 g en las mujeres de ambos grupos. Por su parte, Sheikhazadi et al. en estudio de 1.222 individuos Iraníes, registraron $1.501,6 \mathrm{~g}$ en el hombre y $1.372,2 \mathrm{~g}$ en la mujer, siempre menor que nuestros resultados.

Sheikhazadi et al., señalaron que el peso de este órgano va decreciendo después de la mitad de la vida. En nuestra serie encontramos una leve disminución en los hombres del grupo étnico Mapuche, pero obtuvimos un leve aumento en ambos sexos del grupo NoMapuche, diferente de las mujeres del grupo Mapuche en que sí hubo un aumento significativo del peso del órgano en cuestión, por lo que nuestra concordancia con los autores mencionados es parcial.
Considerando el índice de masa corporal, el peso promedio del hígado, aumenta en los hombres de ambos grupos étnicos en alrededor de un tercio del peso registrado en personas con IMC de 20-25, a diferencia de las mujeres de ambos grupos, en que el peso disminuye levemente. Esta premisa es concordante - en el caso de los hombres - con lo señalado por Sheikhazadi et al., quienes correlacionaron positivamente el peso del hígado con el IMC en ambos sexos. Nuestros resultados en mujeres no concuerdan con estos autores, ya que encontramos un leve decrecimiento en el peso del hígado y la serie presentada por ellos, muestra que al alcanzar un IMC de hasta 25, el incremento es de alrededor de $26 \%$ respecto a individuos con un IMC menor de 20. También se muestra un incremento mayor $(49,2 \%)$ comparando el peso del hígado de un IMC menor a 20 respecto a IMC menor de 39.

Con respecto al bazo, el estudio de Prakash et al. arrojó valores promedios mayores que los de Yi-Suk et al., donde los primeros indicaron un promedio de $149 \mathrm{~g}$ en el hombre y de $153 \mathrm{~g}$ en la mujer, y, los segundos un valor de 115,3 para los hombres y de 99,5 para las mujeres. Los resultados obtenidos en nuestro grupo étnico Mapuche se asemejan bastante a los de estos últimos autores, pero los del grupo No-Mapuche, siendo un poco mayores, son inferiores a los valores de Prakash et al. Por su parte, Sheikhazadi et al., informaron que en iraníes el bazo tiene un peso promedio de $172,1 \mathrm{~g}$ en el hombre y de $166,5 \mathrm{~g}$ en la mujer, bastante mayores a los registrados en nuestra serie.

Los resultados de nuestra serie, en relación a la edad, han mostrado que en los hombres de ambos grupos étnicos y las mujeres NoMapuches, el peso promedio del bazo disminuye alrededor de un $4 \%$, a diferencia de las mujeres del grupo Mapuche en que se incrementa el peso de este órgano en un $18 \%$, no teniendo una explicación para esta diferencia. Los valores informados por Sheikhazadi et al., muestran que el peso del bazo inicialmente aumenta aproximadamente un 7,2\% entre el grupo de 15-24 años al de 25-34 y luego se mantiene hasta 44-54 años, para finalmente alcanzar un aumento de sólo $3,1 \%$ en los 55-64 años.

En relación al índice de masa corporal, el peso del bazo aumenta en los hombres de ambos 
grupos étnicos en alrededor de un $40 \%$, diferente del resultado de Sheikhazadi et al., en que no hubo un aumento del peso de este órgano entre IMC menor a 20 hasta IMC menor a 39. En las mujeres, el peso aumentó en el grupo Mapuche y en las No-Mapuche disminuyó aproximadamente un sexto de su peso original. Los autores mencionados anteriormente, registraron un aumento de hasta $42,9 \%$ considerando el peso del órgano en IMC de menos 20 hasta el de menos 39.

Los riñones de nuestra serie tuvieron pesos semejantes en ambos grupos estudiados, alrededor de $150 \mathrm{~g}$ para el riñón derecho masculino y de $120 \mathrm{~g}$ el femenino, así como valores semejantes en el riñón izquierdo, registrando sí, un peso levemente mayor en el grupo No- Mapuche, que no sobrepasó los $10 \mathrm{~g}$, diferente de lo afirmado por Testut \& Latarjet que además de señalar un peso medio de $170 \mathrm{~g}$, indicaron que el riñón izquierdo es más voluminoso que el derecho, por lo que podría ser de mayor peso. Sheikhazadi et al., registraron un peso promedio para el riñón derecho de $147,9 \mathrm{~g}$ en el hombre y $130,9 \mathrm{~g}$ en la mujer, datos semejantes al obtenido en nuestro estudio. Para el riñón izquierdo, estos autores indicaron que pesa 150,3 $\mathrm{g}$ en el hombre y $135,3 \mathrm{~g}$ en la mujer, valores que concordarían con lo expuesto por Testut \& Latarjet, que señalaron que el riñón izquierdo es más voluminoso que el derecho.

Los pesos informados por Prakash et al., son levemente inferiores a los de nuestra serie en ambos grupos, ya que registraron 136,7 g para el riñón derecho y 132,4 g para el izquierdo en el hombre, y en la mujeres $111,9 \mathrm{~g}$ y 104,2 g, respectivamente. Según Sheikhazadi et al., y Singh et al. (2004) el máximo peso de los riñones se alcanza entre 35 y 44 años y 40 y 50 años, respectivamente. Nuestro resultados concuerdan con estos autores, excepto con el grupo de mujeres de ambos grupos étnicos en que los riñones tienen un peso mayor entre 30 y 39 años.

De acuerdo a Sheikhazadi et al., la mayoría de los órganos alcanza su peso máximo a mitad de la vida, excepto el corazón y la próstata, que van incrementando su peso a través de la vida, premisa que ha corroborado recientemente Olave et al. (2014) respecto al corazón.

Considerando la edad, en ambos sexos del grupo étnico Mapuche, los riñones tienen un leve incremento en su peso promedio. En hombres No- Mapuches estos órganos tienen un incremento de alrededor de $10 \%$ a diferencia de las mujeres en que se mantiene el peso del riñón derecho.

De acuerdo al IMC, el peso promedio de los riñones aumenta en hombres de ambos grupos étnicos, principalmente el riñón derecho. En las mujeres no Mapuches ambos riñones incrementan su peso, principalmente el derecho, a diferencia de las mujeres Mapuches donde el peso se mantiene o disminuye levemente. Sheikhazadi et al., mostraron que el riñón derecho del hombre incrementa su peso en $12,5 \%$, entre IMC de menos 20 hasta IMC de menos 39 y el riñón izquierdo aumenta 7,9\% entre los IMC mencionados; en la mujer, ambos riñones incrementan su peso en $20 \%$ entre estos IMC.

En estudio realizado en individuos vivos, Caucasianos y Afroamericanos, a través de resonancia magnética, He et al., (2009) concluyeron que las personas de más edad tienen una masa menor en órganos como el cerebro, riñones, hígado y bazo, comparado con sujetos jóvenes. De acuerdo a nuestros resultados y considerando que una masa menor de un órgano debería traducirse en un peso menor, concordamos con estos autores parcialmente, ya que sólo el hígado en el hombre Mapuche disminuye en alrededor de un 3\% del peso considerado inicial (personas entre 20 y 29 años), y en el grupo NoMapuche aumenta levemente $(0,6 \%$ en la mujer y $6,4 \%$ en el hombre), así como en la mujer Mapuche que aumenta en alrededor de un sexto de su peso entre la edad de 2029 años a 50-59 años.

Para el caso de los riñones, en hombres y mujeres Mapuche su peso aumentó hasta en 5\% en ambos riñones; en el hombre No-Mapuche el peso de ambos aumentó en alrededor de un $10 \%$, no así en la mujer No-Mapuche en que el peso se mantuvo en el riñón derecho y aumentó en $15 \%$ el izquierdo. Por último, el bazo sí, disminuyó un poco en hombres de ambos grupos étnicos y en las mujeres No-Mapuches.

Ya relacionando con otra de las variables, Prakash et al., concluyeron que el peso del hígado, bazo y riñones fue positivamente correlacionado con el peso del cuerpo. En nuestra serie también aumentaron los pesos de estos órganos en los hombres de ambos grupos étnicos, a medida que aumentó el IMC. Variaciones se encontraron para el caso de las mujeres, ya que el hígado disminuyó levemente en ambos grupos étnicos; los riñones mantuvieron el peso o disminuyó levemente en las mujeres del grupo étnico Mapuche, en cambio aumentó en las mujeres NoMapuche.

Los resultados obtenidos en esta investigación, demuestran la variabilidad del peso de los órganos en estudio, cuando se consideran edad, sexo e índice de masa corporal, comparados con otras poblaciones del mundo, y son un aporte significativo al conocimiento del peso del hígado, bazo y riñones de personas descendientes de dos grupos étnicos del Sur de Chile. 
OLAVE, E.; TORREZ, J. C.; RIQUELME, N.; IBACACHE, L. \& BINVIGNAT, O. Abdominal Organs: weight of the liver, spleen and kidneys in relation to sex, ethnic group, age and body mass index in Chilean individuals. Int. J. Morphol., 32(4):1311-1315, 2014.

SUMMARY: The increase of organ weight may be a sign of disease or pathological abnormalities. Several studies have led to the development of tables with standard weights of different organs in various population groups. Based on this premise, we determined the weight of the liver, spleen and kidneys in two groups of Chilean individuals, Mapuche (M) and non-Mapuche (nM) of both sexes and related the results with age and body mass index (BMI). The average weight of the liver in man in relation to age, slightly increases (nM)) or decreases in small percentage (M). However, in women $(\mathrm{M})$ its weight increased by $22.8 \%$ in relation to BMI, the average weight of the liver increases in males of both ethnic groups in about a third of the weight recorded in people with BMI 20-25; in women of both groups the weight decreases slightly. Spleen weight in relation to age, decreases by about $4 \%$ in men and women from both ethnic (nM) groups, unlike that of Mapuche women (M) where weight increased by $18 \%$. In relation to BMI, spleen weight increased in males of both ethnic groups by about $40 \%$. The kidneys had a slight increase in average weight in both groups. According to BMI, the kidney weights increased in males of both groups. In women (M) the weight is maintained or decreased slightly; in women (nM) both kidneys increase their weight. The data provided in this study will serve as a reference for clinical and surgical anatomy of the abdominal organs of our population.

KEY WORDS: Anatomy; Abdomen; Weight; Liver; Spleen; Kidneys.

\section{REFERENCIAS BIBLIOGRÁFICAS}

Gardner, E.; Gray, D. \& O’Rahilly, R. Anatomía. Estudio por regiones del cuerpo humano. $2^{\mathrm{a}}$ ed. Barcelona, Salvat, 1977.

He, Q.; Heshka, S.; Albu, J.; Boxt, L.; Krasnow, N.; Elia, M. \& Gallagher, D. Smaller organ mass with greater age, except for heart. J. Appl. Physiol. (1985), 106(6):1780-4, 2009.

Molina, D. K. \& DiMaio; V. J. Normal organ weights in men: part II-the brain, lungs, liver, spleen, and kidneys. Am. J. Forensic Med. Pathol., 33(4):368-72, 2012.

Olave, E.; Torrez, J. C.; Riquelme, N.; Ibacache, L. \& Binvignat, O. Weight of the heart and lungs, with regard to sex, ethnic group, age and body mass index in Chilean individuals. Int. $J$. Morphol., 32(3):866-70, 2014.

Prakash, C.; Deopa, D. \& Thakkar, H. K. Study of internal organ weight and its correlation to body weight in Kumaon Region of Uttarakhand. J. Indian Acad. Forensic Med., 35(1):29-32, 2013.

Sheikhazadi, A.; Sadr, S. S.; Ghadyani, M. H.; Taheri, S. K.; Manouchehri, A. A.; Nazparvar, B.; Mehrpour, O. \& Ghorbani, M. Study of the normal internal organ weights in Tehran's population. J. Forensic Leg. Med., 17(2):78-83, 2010.

Singh, D.; Bansal, Y. S.; Sreenivas, M.; Pandey, A. N. \& Tyagi, S. Weights of human organs at autopsy in Chandigarh zone of north-west India. J. Indian Acad. Forensic Med., 26(3):97-9, 2004.

Testut, L. \& Latarjet, A. Tratado de Anatomía Humana. Barcelona, Salvat, 1969.

Williams, R. L.; Warwick, R.; Dyson, M. \& Bannister, L. H. Gray Anatomía. 37a ed. Rio de Janeiro, Guanabara-Koogan, 1995.
Yi-Suk, K.; Deog-Im, K.; Sung Yong, C.; Myoung Hoi, K.; Kyoung Moo, Y.; Han Young, L. \& Seung-Ho, H. Statistical analysis for organ weights in Korean adult autopsies. Korean J. Anat., 42(4):219-24, 2009.

\author{
Dirección para Correspondencia: \\ Dr. Enrique Olave \\ Facultad de Medicina \\ Universidad de La Frontera \\ Casilla 54-D \\ Temuco \\ CHILE
}

Email: enrique.olave@ufrontera.cl

Recibido : 25-05-2014

Aceptado: 30-08-2014 